\title{
Acne Scar Successfully Treated with a Picosecond Laser and Subdermal Minimal Surgery Technique
}

\author{
Ji Yeon Hong ${ }^{1}$ \\ Seong Jun Seo ${ }^{1}$ \\ Kui Young Park ${ }^{1}$ \\ Sang Ju Lee ${ }^{2}$ \\ ${ }^{1}$ Department of Dermatology, Chung-Ang
University College of Medicine, Seoul, Korea
${ }^{2}$ Yonsei Star Skin \& Laser Clinic, Seoul, Korea
}

Received October 27, 2017

Revised October 31, 2017

Accepted November 1, 2017

\section{Correspondence}

Sang Ju Lee

Yonsei Star Skin \& Laser Clinic, 73 Sinchon-ro,

Seodaemun-gu, Seoul 03789, Korea

Tel.: +82-2-332-0023

Fax: +82-2-324-0063

E-mail: drdermadnaver.com

Kui Young Park

Department of Dermatology, Chung-Ang University Hospital, 224-1 Heukseok-dong,

Dongjak-gu, Seoul 06973, Korea

Tel.: +82-2-6299-1525

Fax: +82-2-6299-1718

E-mail: kykyðcaumc.or.kr

(c) Korean Society for Laser Medicine and Surgery

(c) This is an open access article distributed under the terms of the Creative Commons Attribution NonCommercial License (http://creativecommons.org/ licenses/by-nc/4.0) which permits unrestricted noncommercial use, distribution, and reproduction in any medium, provided the original work is properly cited.
Effective therapy of acne scarring remains a major challenge. If severe, it can be psychologically debilitating, leading to low self-esteem and passive mindset. In this study, we report a good cosmetic outcome after a combination treatment of pneumatic needless microjet injection and picosecond laser, incorporating a novel holographic beam splitter handpiece. Our results support the use of a fractional neodymium-doped yttrium-aluminum-garnet, picosecond-domain laser as an effective and safe treatment modality to treat facial acne scarring.

\section{Key words}

Acne scar; Picosecond laser; Subdermal minimal surgery technique 


\section{INTRODUCTION}

Acne and subsequent scarring, as well as residual erythema and postinflammatory hyperpigmentation often cause severe effects on cosmetic and psychosocial aspects. ${ }^{1}$ Especially, many patients are willing to treat acne scars because the scars usually last for a long time and are reluctant to camouflage with casual makeup. Current therapeutic modalities include ablative or nonablative laser skin resurfacing, chemical peeling, microdermabrasion, punch elevation, punch graft, subcision, filler injection and dermal rolling. ${ }^{2}$ However, effective therapy of acne scarring remains a major challenge. Herein, we report cosmetic improvements in facial acne scars after treating with subdermal pneumatic injection and picosecond laser incorporating a holographic optic.

\section{CASE REPORT}

A 23-year-old healthy female presented with multiple pitting acne scars on her cheeks (Fig. 1A). To treat her pitting scars, we first tried subdermal minimal surgery technique with pneumatic needleless microjet injector (INNOJECTOR $^{\text {TM }}$; Amore Pacific, Seoul, Koreal. The accelerated jet of normal saline pierces into subdermal level via small entry point on the epidermis, with the volume of ejection per each time as $0.15 \mathrm{ml}$. After 1 month, the scars showed mild improvement, but the patient wanted more dramatic change. Therefore, 532-nm neodymium-doped yttrium-aluminium-garnet (Nd:YAG), picosecond-domain laser system (PicoWay ${ }^{\circledR}$; Syneron-Candela Corporation, Wayland, MA, USA), with a holographic beam-splitting optic (PicoWay Resolve ${ }^{\circledR}$; Syneron-Candela Corporation) was recommended to the patient. No topical anesthesia was applied before the laser treatment. Three laser passes were administered sequentially to the cosmetic unit in a painting mode with the energy of $2.6 \mathrm{~mJ} /$ microbeam in a frequency of 6- $\mathrm{Hz}$ at $532-\mathrm{nm}$, until desired endpoints of mild erythema and edema were achieved. We repeated the procedure with the same parameters twice at 1-month intervals. After the sessions were completed, the scars were significantly improved and the patient was very satisfied with the cosmetic outcome (Fig. 1B). No severe adverse effects were detected except for instant erythema and procedural discomfort, which resolved within a few hours.

\section{DISCUSSION}

Although dermatologists have a potent arsenal of treatments for inflammatory acne lesions, the scarring residue of acne still remains hard to overcome. ${ }^{2}$ The newly developed pneumatic needleless microjet injector emits a high-speed jet up to $180 \mathrm{~m} / \mathrm{sec}$ through a $0.1-\mathrm{mm}$ nozzle hole, permeating the epidermis and delivering target material intradermally to a controlled depth. ${ }^{3}$ It induces dermal microtrauma which in turn, stretches fibroblasts, stimulates growth factors and inhibit collagen breakdown. ${ }^{4}$ Kim et al. ${ }^{5}$ reported the effectiveness of pneumatic device for the treatment of depressed scars of the forehead caused by herpes zoster.

$Q$-switched laser that generates picosecond-domain pulses was recently introduced and has already demonstrated its effectiveness at removing unwanted tattoos. ${ }^{6}$ Furthermore, an innovative holographic fractional handpiece for the picosecond laser has been developed to produce fractional wavelengths of $532 \mathrm{~nm}$ and 1,064 nm. The new lens array helps deliver all the laser energy into highly focused areas in a square grid. Therefore, the picosecond laser can exploit laser-induced optical breakdown of dermal tissue to achieve skin rejuvenation and treat acne scars. ${ }^{7}$

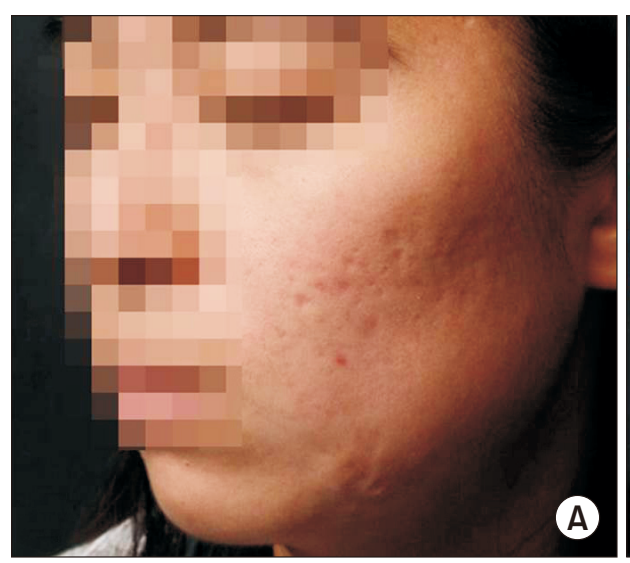

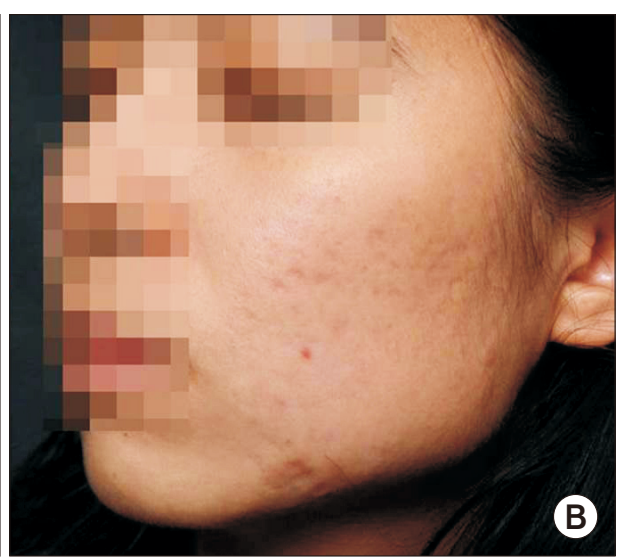

Fig. 1. (A) Before treatment: remarkable pitting scars on the cheek (B) significant improvement of the skin texture after 3 months. 
Good cosmetic results were produced by combination therapy using picosecond-domain pulses and subdermal minimal surgery technique for atrophic acne scars. This case demonstrates that the picosecond Nd:YAG laser with a holographic beam splitter has its potential in treating various kinds of depressed scars. To confirm optimal parameters, controlled studies with a larger population are needed.

\section{REFERENCES}

1. Hazarika N, Archana M. The psychosocial impact of acne vulgaris. Indian J Dermatol 2016;61:515-20.

2. Lipper GM, Perez M. Nonablative acne scar reduction after a series of treatments with a short-pulsed 1,064-nm neodymium:YAG laser. Dermatol Surg 2006;32:998-1006.

3. Seok J, Choi SY, Park KY, Jang JH, Bae JH, Kim BJ, et al. Depressed scar after filler injection successfully treated with pneumatic needleless injector and radiofrequency device. Dermatol Ther 2016;29:45-7.

4. Levenberg A, Halachmi S, Arad-Cohen A, Ad-El D, Cassuto D, Lapidoth M. Clinical results of skin remodeling using a novel pneumatic technology. Int J Dermatol 2010;49:1432-9.

5. Kim BJ, Yoo KH, Kim MN. Successful treatment of depressed scars of the forehead secondary to herpes zoster using subdermal minimal surgery technology. Dermatol Surg 2009;35:143940.

6. Bernstein EF, Schomacker KT, Basilavecchio LD, Plugis JM, Bhawalkar JD. A novel dual-wavelength, Nd:YAG, picoseconddomain laser safely and effectively removes multicolor tattoos. Lasers Surg Med 2015;47:542-8.

7. Habbema L, Verhagen R, Van Hal R, Liu Y, Varghese B. Minimally invasive non-thermal laser technology using laser-induced optical breakdown for skin rejuvenation. J Biophotonics 2012;5:194-9. 